\title{
The Effect of Asperity Flattening During Cyclic Normal Loading of a Rough Spherical Contact
}

\author{
Longqiu Li • Andrey Ovcharenko · Izhak Etsion • \\ Frank Talke
}

Received: 20 April 2010/ Accepted: 13 July 2010/Published online: 25 July 2010

(C) The Author(s) 2010. This article is published with open access at Springerlink.com

\begin{abstract}
The effect of asperity flattening of a rough spherical contact during cyclic loading is investigated experimentally. Two types of surfaces are examined; the first is an "as-manufactured" isotropic surface and the second a smooth "laser-polished" surface. Both the surfaces exhibit a large amount of hysteresis of the loaddisplacement curve during the first load-unload cycles. This hysteresis is found to decrease as a function of the number of load cycles. A comparison of the experimental results with results obtained from a numerical model for a rough spherical contact shows good correlation. The model shows that for rough surfaces the total displacement is a function of the contacting asperities while for smooth surfaces the main contribution comes from the bulk displacement.
\end{abstract}

Keywords Contact mechanics - Spherical contact .

Rough surface $\cdot$ Cyclic load-unload

$\begin{array}{ll}\text { Abbreviations } \\ a & \text { Radius of contact } \\ E & \text { Young's modulus } \\ H & \text { Hardness } \\ h_{0} & \text { Minimum separation } \\ h_{0}^{*} & \text { Dimensionless minimum separation } h_{0} / \sigma\end{array}$

\section{Li $(\square)$}

Harbin Institute of Technology, Harbin 150001, China

e-mail: longqiuli@gmail.com

L. Li · A. Ovcharenko · F. Talke

University of California San Diego, La Jolla,

CA 92093-0401, USA

I. Etsion

Technion-Israel Institute of Technology, Haifa 32000, Israel $h_{1}^{*} \quad$ Dimensionless displacement of the contacting asperities $h_{1} / \sigma$

$L \quad$ Spherical shell height

$P \quad$ Normal load

$P_{\mathrm{c}} \quad$ Critical normal load

$P^{*} \quad$ Dimensionless normal load $P / P_{\mathrm{c}}$

$R \quad$ Radius of the spherical shell

$Y \quad$ Yield strength

$t \quad$ Spherical shell thickness

$\beta \quad$ Roughness parameter $\eta \rho \sigma$

$\gamma \quad$ Total displacement of the rough spherical contact

$\eta \quad$ Area density of asperities

$\rho \quad$ Asperity tip radius of curvature

$\sigma \quad$ Standard deviation of surface heights

$\gamma^{*} \quad$ Dimensionless total displacement $\gamma / \sigma$

$\gamma_{\max }$ Maximum displacement

$\gamma_{\text {res }} \quad$ Residual displacement

$\lambda \quad$ Shell parameter

$v \quad$ Poison's ratio

$\sigma_{\mathrm{s}} \quad$ Standard deviation of asperity heights

$\Psi \quad$ Plasticity index

$\omega \quad$ Displacement of the sphere bulk

$\omega^{*} \quad$ Dimensionless displacement $\omega / \sigma$

$\omega_{\mathrm{c}} \quad$ Critical interference

$\omega_{\mathrm{c}}^{*} \quad$ Dimensionless critical interference $\omega_{\mathrm{c}} / \sigma$

\section{Introduction}

Elastic-plastic contacts of rough spherical surfaces can occur in many engineering applications such as MEMS micro-switches [1], AFM systems [2], or magnetic storage devices [3, 4]. In these applications, loading and unloading of the contacting surfaces occur in a repetitive, cyclic manner. Cyclic loading of contacts can generate wear 


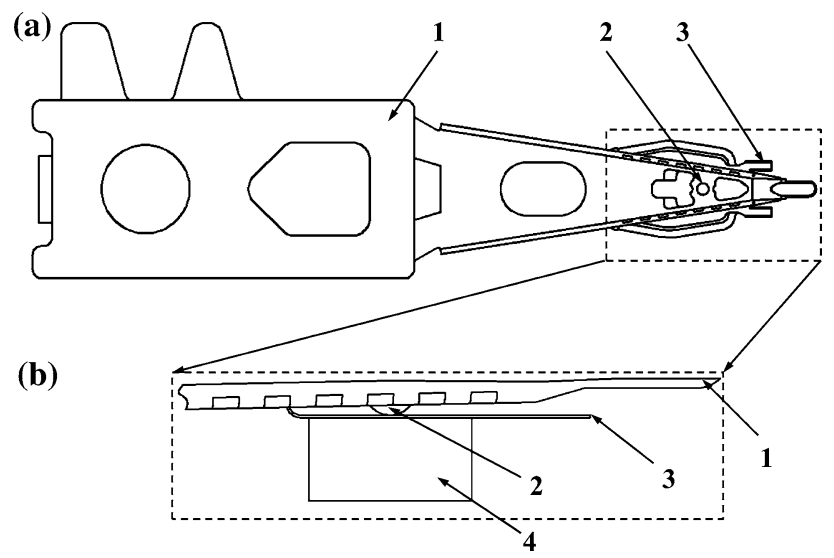

Fig. 1 Schematic diagram and enlarged detail of a hard disk drive suspension assembly: a top view and $\mathbf{b}$ side view of dimple/gimbal zone. Shown in the figure are the 1 suspension, 2 dimple, 3 gimbal, and 4 slider

particles which can significantly shorten the lifetime of the devices.

A typical example of a rough spherical contact is the dimple/gimbal interface in a hard disk drive (HDD) suspension. There, a suspension spring features a spherical protrusion (dimple), which is loaded against a flat gimbal that carries the magnetic recording slider (Fig. 1). During operation of a disk drive, cyclic loading of the gimbal and the dimple occurs which can lead to the formation of wear particles [5]. Wear particles are undesirable since they can cause failure of the head/disk interface.

Several numerical models can be found in the literature for the contact of a smooth sphere and a rigid flat (see references [6-9]). These models, which can represent a single asperity of a rough surface, are used in statistical descriptions of spherical contact of rough surfaces with either Gaussian distribution [10] or other statistical distribution of asperity heights [11, 12].

A number of experimental studies of a single spherical contact also exist in the literature [13-18]. Parker and Hatch [13] investigated hemispherical specimens of indium and lead pressed against a glass flat under elastic-plastic or purely plastic deformation. Chaudhri and Yoffe [14] investigated the contact area of steel and WC balls pressed against fused silica, soda-lime glass and sapphire flats in purely elastic deformation. The measured contact radii were compared with the Hertz theory and good agreement was obtained for a steel ball in contact with a glass flat. Ovcharenko et al. [15] investigated the contact of copper and stainless steel spheres of different diameters that were pressed against a sapphire flat during loading, unloading, and cyclic load-unload in the elastic-plastic regime of deformations. An in situ, optical technique was used to observe the evolution of the contact area. Good agreement was found between experimental [15] and theoretical [6, 8, 9] results for the contact area and mean contact pressure.

Several experimental investigations have been conducted that were specifically aimed at studying contact of rough spherical surfaces [19] or flattening of the asperities of rough surfaces [20]. Jamari and Schipper [19] performed an experimental investigation into the deformation behavior of a smooth sphere and a real rough surface. Their experimental results were in good agreement with theoretical prediction. They also observed that the total deformation consisted of asperity and bulk contributions. Lo et al. [20] conducted a series of experiments using aluminum strips to investigate the evolvement of surface roughness and the resistance of surface roughness to flattening in forming processes.

Several theoretical models have been published concerning unloading and cyclic load-unload of a deformable single sphere or a rough spherical contact [21-25]. Only two published experimental investigations $[15,26]$ seem to have been performed concerning the contact area during unloading and cyclic load-unload. Qian et al. [26] used a nano-indenter to perform cyclic load-unload tests (which they termed radial nano-fretting) of a Berkovich diamond tip against typical structural materials used in MEMS technology. Their experimental results exhibit hysteretic behavior of force versus displacement curve. However, the residual deformation decreases quickly to zero, and both the contact stiffness and the projected area of the indents approach a constant value, after the first cycle.

As can be seen from the above literature review, only a few experimental studies exist in the literature on surface roughness effects of rough spherical contacts. Furthermore, no experimental studies are present for asperity flattening during multiple loading-unloading cycles of rough spherical contact. It is, therefore, the main goal of the present investigation to focus on this missing information by studying experimentally the effect of surface roughness on asperity flattening during cyclic loading of a rough spherical contact. Such studies could be useful, for example, in understanding the generation of wear particles in the dimple/gimbal interface of a hard disk drive, or other similar applications.

\section{Experimental Details}

Cyclic load-unload tests were performed using spherical dimples of a hard disk drive suspension (see Fig. 1). These dimples were cut out from the hard disk drive suspension (NHK International Corp., USA) as shown in Fig. 2. They were made of stainless steel (SST304) and had the form of a spherical shell (see Fig. 2b) with an outer radius $R=200 \mu \mathrm{m}$, a height $L=80 \mu \mathrm{m}$ and a wall thickness 
(a)

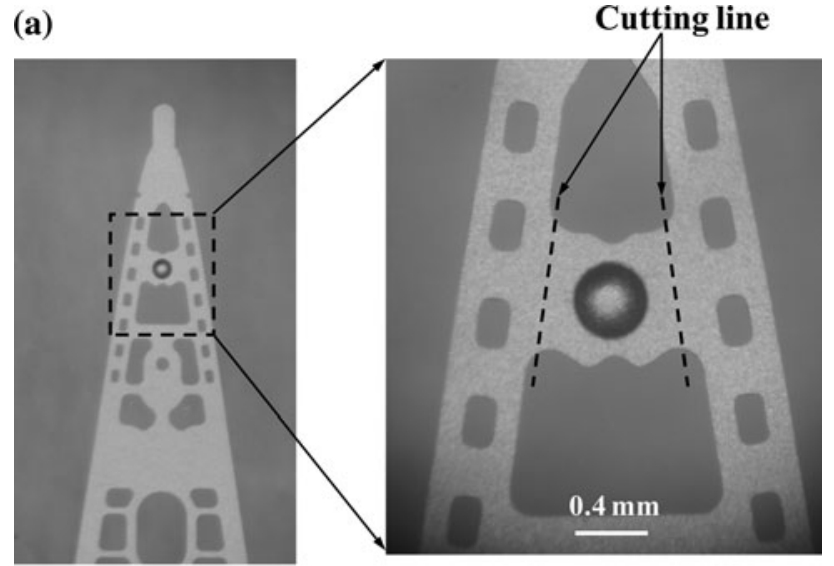

(b)

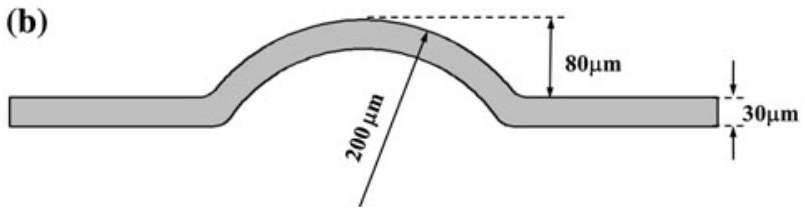

Fig. 2 a Photograph of hard disk drive suspension showing the protruding dimple. The dashed lines indicate where the suspension is cut for the preparation of the dimple specimen; b Schematic crosssection of the dimple showing its dimensions

$t=30 \mu \mathrm{m}$. Figure 3 presents a schematic diagram of the test set-up. Each spherical dimple was glued, using epoxy (Devcon, USA), on the bottom surface of the plastic holder (see Fig. 4), and pressed repeatedly against the rigid sapphire flat shown schematically in Fig. 3. Figure 5 shows a photograph of the test rig, which consists of a modified nano-indenter (Hysitron Inc., USA), where the conventional hard diamond tip was replaced by the dimple specimen. The normal load (limited to not more than $9 \mathrm{mN}$ ) and the corresponding displacement of the dimple were measured using the transducer of the nano-indenter.

Two different types of dimples, a so-called "rough dimple" and a smooth "laser-polished dimple," were used for the tests. The dimple surface roughness was measured using an atomic force microscope (AFM, Pacific Nanotechnology, USA). The arithmetic average surface roughness values $(\mathrm{Ra})$ obtained with a scan size of $10 \times 10 \mu \mathrm{m}$ and a sampling interval of $0.69 \mu \mathrm{m}$ were approximately $85 \pm 5$ and $25 \pm 4 \mathrm{~nm}$ for the rough and the smooth dimples, respectively. The Young's modulus $E$ and the hardness $H$ of the dimples were measured with the nanoindenter using a conventional diamond tip. The yield strength $Y$ was calculated from the following equation (see reference [27]):

$\frac{H}{Y}=0.201 \ln \left(\frac{E}{Y}\right)+1.685$

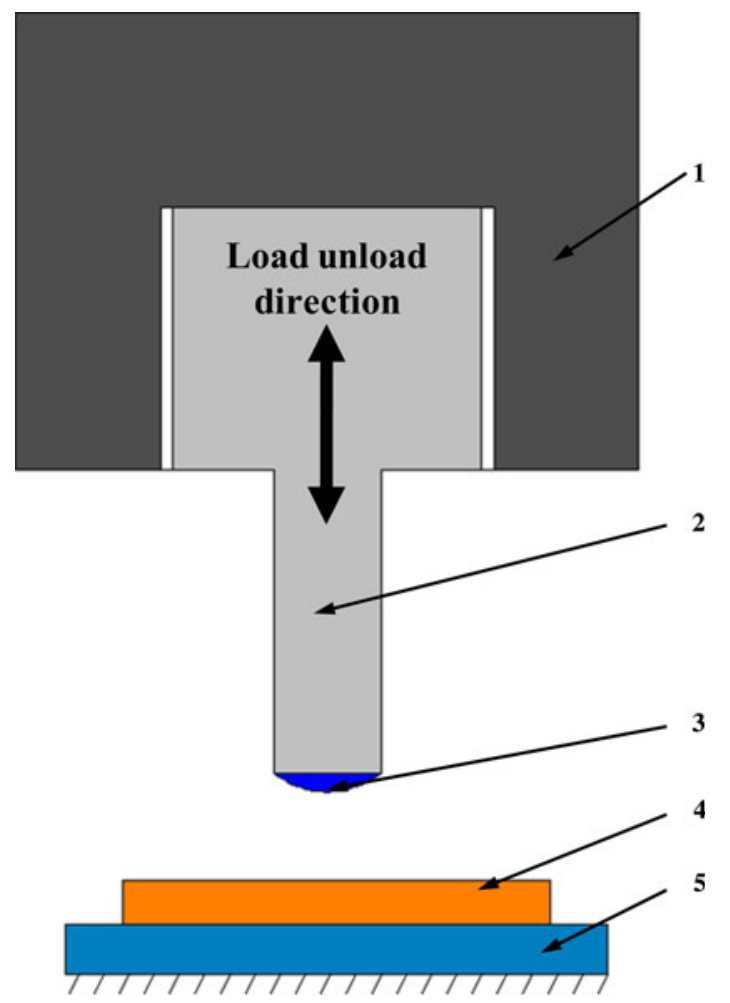

Fig. 3 Schematic representation of the experimental setup; 1 transducer, 2 plastic holder, 3 dimple specimen, 4 rigid flat, and 5 fixed stage

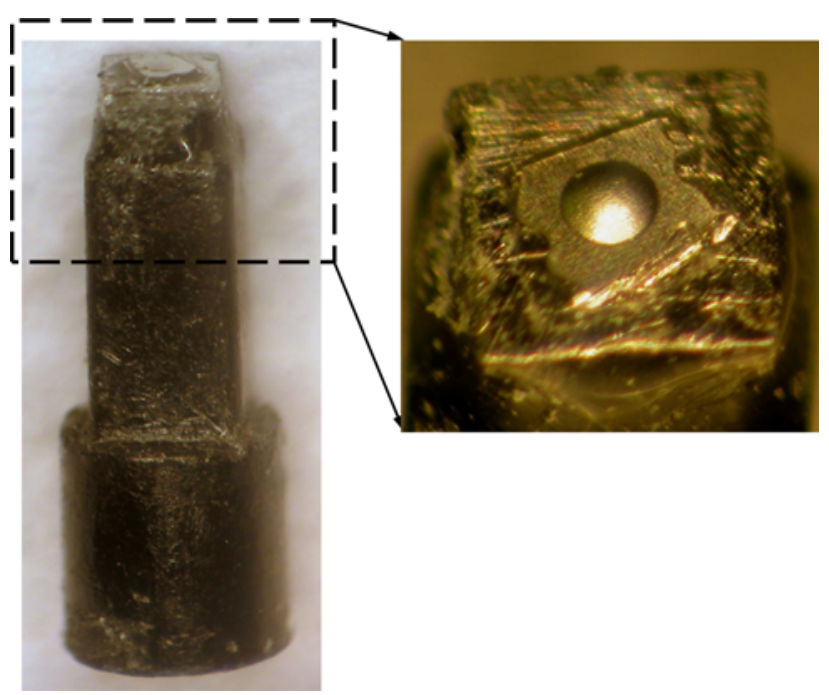

Fig. 4 Photograph of the plastic holder with a dimple glued to its end

The mechanical properties of the two dimple types and the sapphire flat are summarized in Table 1.

All experiments were carried out at room temperature of $20-25{ }^{\circ} \mathrm{C}$ and relative humidity of $40-60 \%$. Each experiment was performed on a new dimple. Both the dimple and the sapphire surfaces were cleaned by acetone prior to 

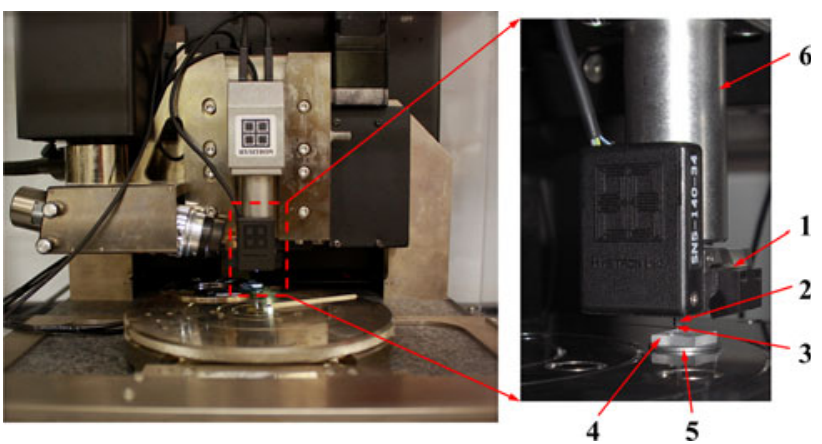

Fig. 5 Photograph of the test rig assembly: 1 transducer, 2 plastic holder, 3 dimple, 4 rigid sapphire flat, 5 stage, and 6 nano-indenter

testing using an Ultrasonic cleaner (Cole-Parmer, USA). All experiments were performed under dry condition. Each load-unload cycle was completed in $10 \mathrm{~s}$ ( $5 \mathrm{~s}$ for loading and $5 \mathrm{~s}$ for unloading).

The surface roughness of each tested dimple was measured both before and after the test to identify asperity flattening.

\section{Experimental Results}

Figure $6 \mathrm{a}$ and $\mathrm{b}$ presents typical experimental results of the applied normal load $P$ versus the measured displacement $\gamma$, for rough and smooth (laser-polished) dimples, respectively. The results are shown for the 1st, 2nd, 5th, and 50th cycle of load-unload. In every cycle, the maximum normal load was set to $P_{\max }=9 \mathrm{mN}$. Each load-unload cycle starts at the origin of the coordinate system where both the load and displacement are zero. Immediately after the engagement of the dimple surface and the rigid sapphire flat the load is increased gradually until it reaches its predefined maximum value of $P_{\max }=9 \mathrm{mN}$. At this point, a corresponding maximum displacement $\gamma_{\max }$ is obtained and the unloading starts. The load is gradually reduced to zero and the cycle ends. As observed in Fig. 6, the various loaddisplacement loops show a hysteretic behavior with a corresponding residual displacement $\gamma_{\text {res }}$ at the end of each unloading. The first load-unload cycle exhibits a substantial larger hysteresis and, hence, more plastic deformation compared to any of the subsequent cycles. These subsequent cycles exhibit a tendency to "elastic shakedown" [28] with decreasing dissipated energy (the area of
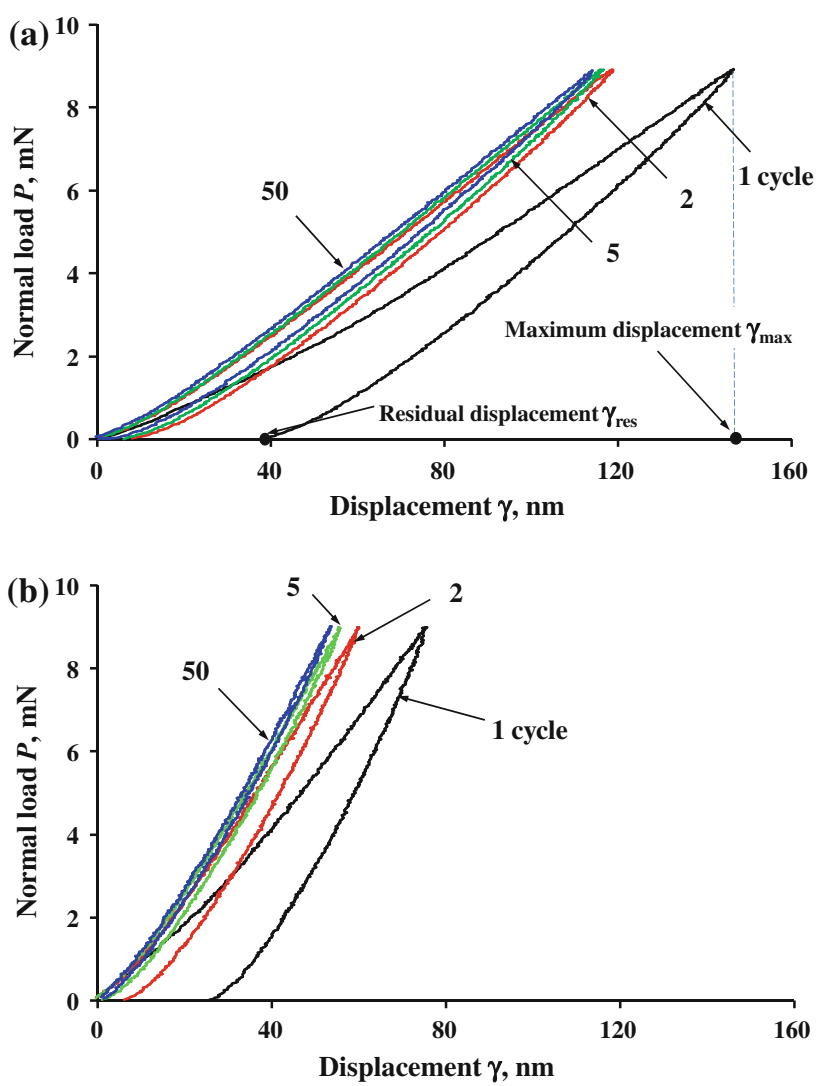

Fig. 6 Typical test results of load displacement loops for various load-unload cycles; a rough dimple and b laser-polished dimple

the hysteretic load-displacement loop of each cycle), as well as increasing stiffness of the contact with increasing number of cycles. This is similar to the behavior observed in reference [26] using a Berkovich diamond tip against typical structural materials. In the rough dimple case (Fig. 6a), the first loading cycle exhibits a maximum displacement of $\gamma_{\max }=146 \mathrm{~nm}$ and a residual displacement of $\gamma_{\text {res }}=40 \mathrm{~nm}$ compared to only 76 and $26 \mathrm{~nm}$, respectively, for the laser-polished dimple case (Fig. 6b). It can also be seen from Fig. 6 that at each load-unload cycle, the area of the hysteretic load-displacement loop in the rough dimple case is larger than the corresponding area in the laser-polished dimple case. This difference clearly indicates that less energy is dissipated in the latter case, as would be expected for smoother and hence, more elastic contacts.

Table 1 Mechanical properties of typical "rough" and "laser-polished" dimples, and of the sapphire flat

\begin{tabular}{|c|c|c|c|c|c|c|c|}
\hline Specimen & Material & $H[\mathrm{GPa}]$ & $Y[\mathrm{GPa}]$ & $v$ & $E[\mathrm{GPa}]$ & $P_{\mathrm{c}}[\mathrm{mN}]$ & $\omega_{\mathrm{c}}[\mathrm{nm}]$ \\
\hline Rough dimple & Stainless steel 304 & 4.31 & 1.68 & $0.31^{\mathrm{a}}$ & 181 & 212 & 186 \\
\hline Laser-polished dimple & Stainless steel 304 & 3.7 & 1.42 & $0.31^{\mathrm{a}}$ & 181 & 128 & 133 \\
\hline Rigid flat & Sapphire & $19^{\mathrm{a}}$ & $2.95^{\mathrm{a}}$ & $0.27^{\mathrm{a}}$ & $435^{\mathrm{a}}$ & - & - \\
\hline
\end{tabular}

a Values obtained from the literature (www.matweb.com, www.gavish.com) 


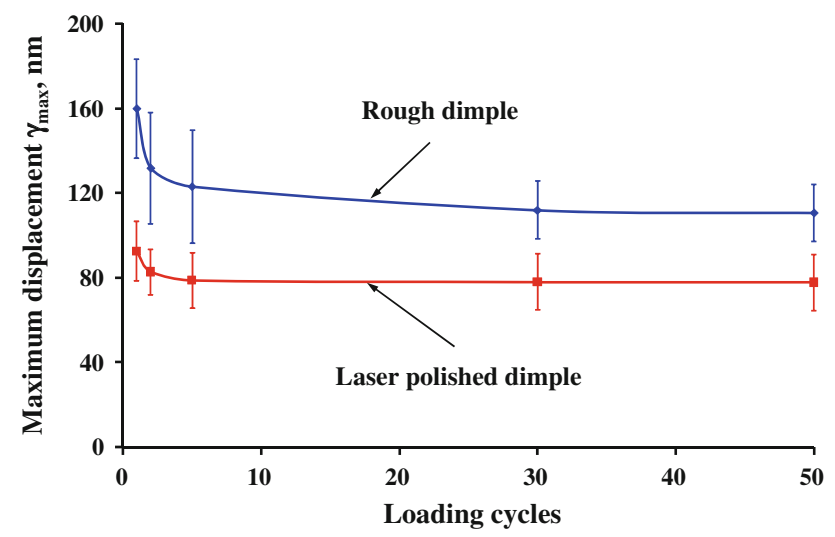

Fig. 7 Average and standard deviation values of the maximum displacement $\gamma_{\max }$ at normal load $P=9 \mathrm{mN}$ vs. the number of loadunload cycles for rough and laser-polished dimples

Figure 7 shows the average and standard deviation values of the maximum displacement $\gamma_{\max }$ versus the number of the load-unload cycles for both the rough and the laser-polished dimples. The results shown in Fig. 7 were obtained from at least four repetitions of tests like those shown in Fig. 6. As can be seen the average value of maximum displacement $\gamma_{\max }$ in both cases decreases with increasing number of load-unload cycles, and approaches a constant value approximately after 5 cycles. The average value of $\gamma_{\max }$ for the smooth dimple is approximately $60 \%$ smaller than that for the rough one.

Figure 8 presents the average and standard deviation values (obtained from at least four test repetitions) of the residual displacement $\gamma_{\text {res }}$ versus the number of the loadunload cycles for the two types of dimples. The average value of $\gamma_{\text {res }}$ decreases sharply during the first five cycles approaching a constant value close to zero, i.e., the contact becomes elastic. Similar to the maximum displacement, the average value of the residual displacement $\gamma_{\text {res }}$ of the laserpolished dimple is nearly 55\% smaller than that of the rough dimple.

The behavior shown in Figs. 6, 7, and 8 indicates that the largest plastic deformation of the spherical dimple occurs at the first load-unload cycle, and that the deformation at subsequent load-unload cycles becomes smaller and smaller, with less and less plastic deformation. This so-called "elastic shakedown" appears to be the result of asperity flattening in the contact zone. It seems justifiable to postulate that this phenomenon is related to a reduction in the plasticity index [29] of the contact zone. In order to verify this hypothesis, the surface roughness was measured at the end of each test and compared with the surface roughness before testing. Figure 9 presents typical AFM images of such measurements for a rough dimple before testing (Fig. 9a) and after 200 load-unload cycles (Fig. 9b), showing the flattened asperities within the

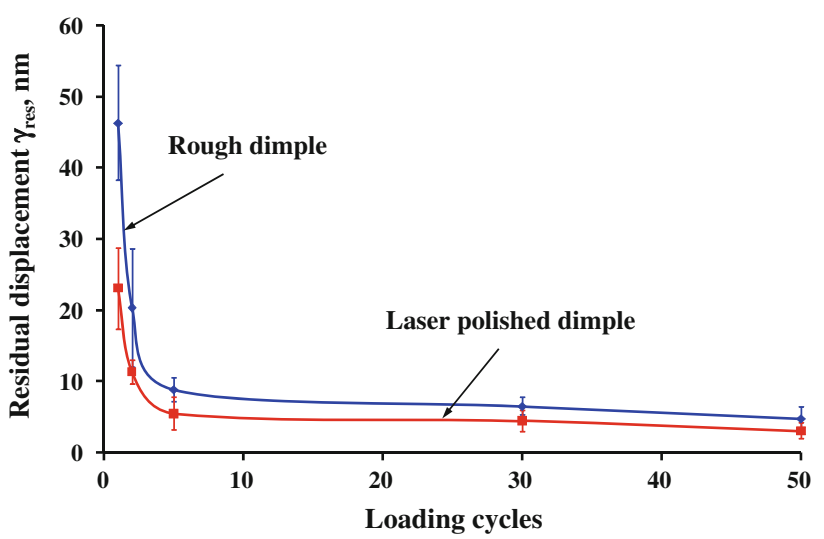

Fig. 8 Average and standard deviation values of the residual displacement $\gamma_{\text {res }}$ vs. the number of load-unload cycles for rough and laser-polished dimples after complete unloading from normal load $P=9 \mathrm{mN}$
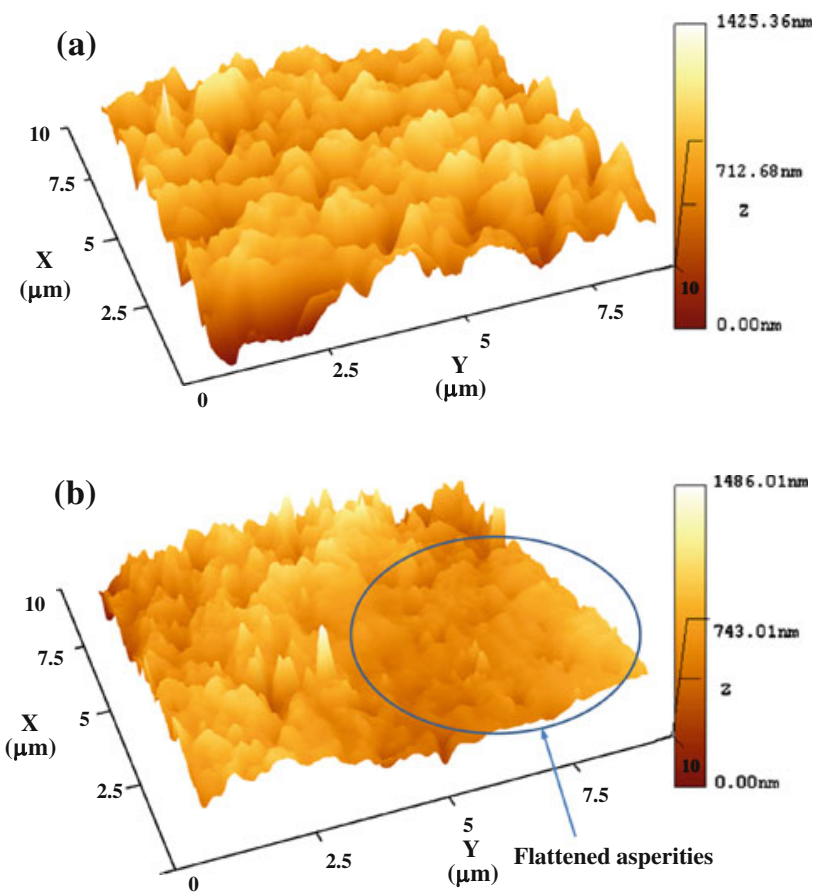

Fig. 9 AFM images of surface roughness of a rough dimple: a before testing and b after 200 load-unload cycles, showing the flattened asperities within the encircled portion of the contact area

encircled contact area. We observe clearly that flattening of the contacting asperities occurs as a function of repeated load-unload cycles.

\section{Comparison Between Experimental and Theoretical Results}

The bulk of the dimple sample used in this study is a spherical shell that can be modeled as shown in Fig. 10a. 

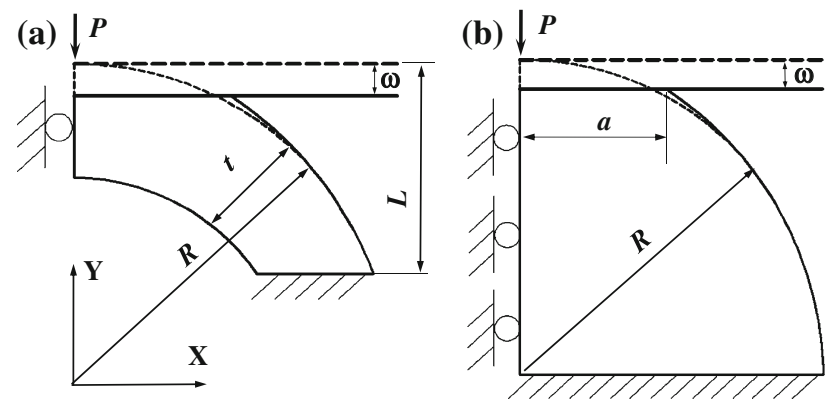

Fig. 10 Schematic model diagrams for: a a spherical shell, and $\mathbf{b}$ a solid sphere, in contact with a rigid flat

The contact of such shell (in elastic deformation) is controlled by its shell parameter $\lambda$ given by [30]:

$\lambda=\log \left(\frac{t}{R}\left(\frac{E}{Y}\right)^{0.886}\right)$

If the shell parameter is $\lambda>0.75$, the solution of the elastic contact problem of such a spherical shell approaches that of a solid sphere with the same radius $R$ (see Fig 10b). From the values presented in Table 1 and the geometry of the dimples used in the present study, Eq. 2 yields values of the shell parameter $\lambda$ about 1 for both dimple types. Therefore, the following analysis for the rough dimple contact behavior will be based on a rough solid sphere contact model presented in reference [31]. The critical load $P_{\mathrm{c}}$ at yield inception can be obtained from Eq. 3 below [8]:

$P_{\mathrm{c}}=\frac{\pi^{3}}{6} C_{\mathrm{v}}^{3} Y\left(R\left(1-v^{2}\right) \frac{Y}{E}\right)^{2}$

where $C_{\mathrm{v}}=1.234+1.256 \mathrm{v}$. The values of $P_{\mathrm{c}}$ for the rough and laser-polished dimples are given in Table 1. These values are much higher than the present maximum load of $9 \mathrm{mN}$. Hence, the bulk of the dimples under examination is elastically deformed and can be analyzed as a solid sphere in elastic contact.

Figure 11 presents an equivalent rough spherical contact model that was studied theoretically by $\mathrm{Li}$ et al. [31]. In reference [31], a model was developed for the effect of the dimensionless normal load $P^{*}$, the dimensionless critical interference $\omega_{\mathrm{c}}^{*}$ and the plasticity index $\psi$, on the individual contributions of the sphere bulk and the asperities to the total displacement of a rough spherical contact. In this model, the total dimensionless displacement $\gamma^{*}=\gamma / \sigma$ of the rough spherical contact is the sum of two displacement components: $\omega^{*}=\omega / \sigma$ (contributed by the sphere bulk alone), and $h_{1}^{*}=h_{1} / \sigma$ (contributed by the contacting asperities). Hence,

$\gamma^{*}=\frac{\gamma}{\sigma}=\omega^{*}+h_{1}^{*}$

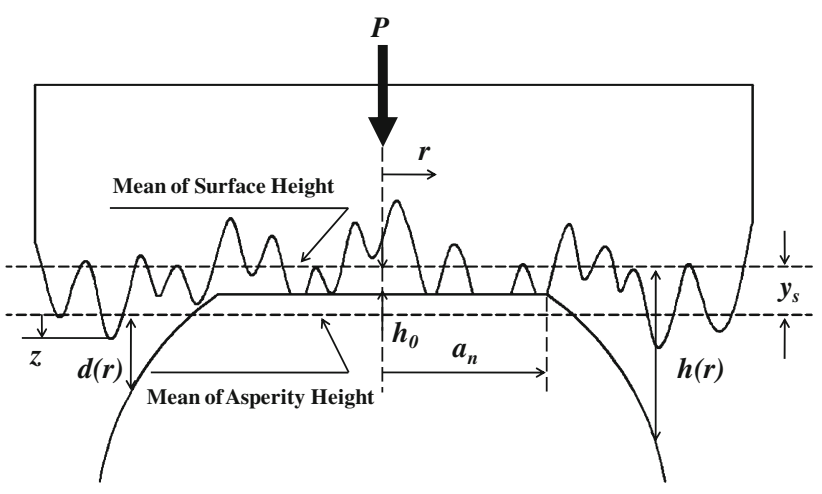

Fig. 11 Schematic diagram of rough spherical contact model [31] showing an equivalent rough flat and a smooth sphere

The plasticity index $\psi$, which affects $h_{1}^{*}$ has the form $[10,31]$ :

$\psi=\frac{2 E}{C_{\mathrm{v}} \pi\left(1-v^{2}\right) Y}\left(\frac{\sigma_{\mathrm{s}}}{\rho}\right)^{0.5}$

The maximum normal load of $9 \mathrm{mN}$ in the present experiments is two orders of magnitude less than the critical normal load $P_{\mathrm{c}}$ (see Table 1). As shown in [31], the dimensionless displacement of the bulk of the sphere for $P^{*} \leq 1$, is:

$\omega^{*}=\omega_{\mathrm{c}}^{*} P^{* 2 / 3}$

where the dimensional critical interference of the sphere $\omega_{\mathrm{c}}=\sigma \omega_{\mathrm{c}}^{*}$ is:

$\omega_{\mathrm{c}}=\left(\frac{C_{\mathrm{v}} \pi\left(1-v^{2}\right) Y}{2 E}\right)^{2} R$

The values of $\omega_{\mathrm{c}}$ for the rough and laser-polished dimples are given in Table 1 .

A dimensionless transition load $P_{\mathrm{t}}^{*}$ was found in reference [31] as a function of $\omega_{\mathrm{c}}^{*}$ and $\psi$. At this transition load, the displacement $h_{1}^{*}$ of the asperities equals the displacement $\omega^{*}$ of the bulk of the sphere. If the value of $P^{*}$ is much smaller than $P_{\mathrm{t}}^{*}$, the contribution of the asperities to the total displacement is dominant. On the other hand, if $P^{*}$ is much larger than $P_{\mathrm{t}}^{*}$ roughness effect is negligible.

To compare the present experimental measurements with the theoretical results of reference [31], the roughness parameters $\rho, \sigma$, and $\sigma_{\mathrm{s}}$ were calculated from the surface roughness measurements of the dimples using the procedure described in [32]. It should be noted here that the measured roughness parameters and, hence, the plasticity index $\psi$ depend on the scan size and the sampling interval of the measurement [33]. For this reason, various scan sizes ranging from $2.5 \times 2.5$ to $50 \times 50 \mu \mathrm{m}$ and various sampling intervals from 0.039 to $1.17 \mu \mathrm{m}$ were used. The 
resulting calculated plasticity indices before testing varied from 3.2 to 30 for the rough dimple and from 0.4 to 12 for the laser-polished dimple. We observed that the best correlation with the theoretical prediction, for both the rough and the laser-polished dimple corresponds to $\psi$ values that resulted from a scan size of $10 \times 10 \mu \mathrm{m}$ and a sampling interval of $0.69 \mu \mathrm{m}$. The measured roughness parameters for the rough dimple under this scanning condition were: $\rho=8.30 \mu \mathrm{m}$, and $\sigma \approx \sigma_{\mathrm{s}}=90 \mathrm{~nm}$. These roughness parameters result in: $\psi=3, \omega_{\mathrm{c}}^{*}=2.07$, and $P_{\mathrm{t}}^{*}=3.03$. For the laser-polished dimple, the corresponding values were: $\rho=12.50 \mu \mathrm{m}, \sigma \approx \sigma_{\mathrm{s}}=30 \mathrm{~nm}, \psi=2.5, \omega_{\mathrm{c}}^{*}=$ 4.43, and $P_{\mathrm{t}}^{*}=0.25$.

Figure 12 shows the dimensionless normal load $P^{*}$ versus the dimensionless displacement $\gamma / \sigma$ for the rough dimple during the first loading. As can be seen, for the actual $P^{*}$ values of the test, which are less than 0.04 , the contribution of the contacting asperities to the total displacement is much larger than that of the bulk of the sphere. This would be expected for dimensionless loads much smaller than the transition load $P_{\mathrm{t}}^{*}=3.03$ [31]. For $\psi=3$ and $\omega_{\mathrm{c}}^{*}=2.07$ good agreement is observed between the theoretical and experimental results of the total displacement. The somewhat larger experimental displacement compared to the theoretical one appears to be related to the compliance present in the experimental system but not in the model.

Figure 13 shows a similar comparison of the experimental and theoretical results, during the first loading, for the laser-polished dimple when $\psi=2.5$ and $\omega_{\mathrm{c}}^{*}=4.43$. Similar to Fig. 12, good correlation is observed between the experimental and theoretical results for the total displacement. The transition load $P_{\mathrm{t}}^{*}=0.25$ of the laserpolished dimple is still larger than the actual $P^{*}$ values of the test but not as much as the $P_{\mathrm{t}}^{*}=3.03$ of the rough

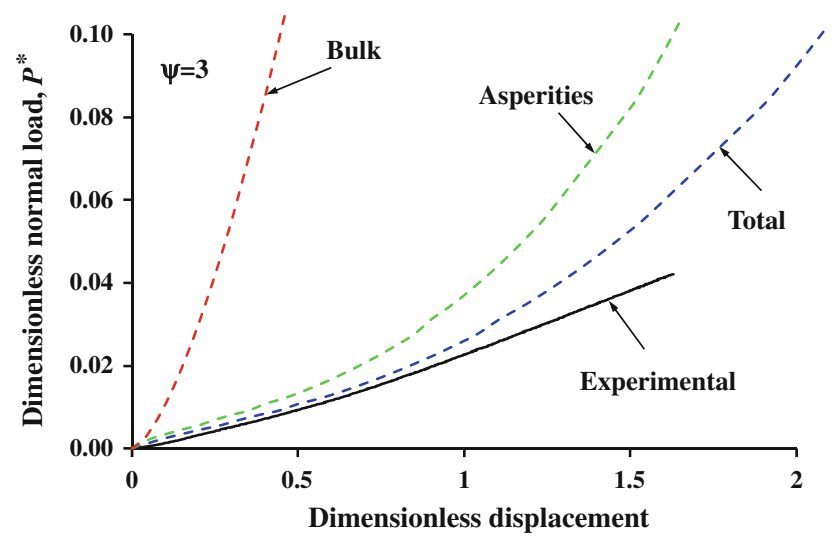

Fig. 12 A comparison of experimental results (solid line) and theoretical predictions (dashed lines $\psi=3, \omega_{\mathrm{c}}^{*}=2.07, P_{\mathrm{t}}^{*}=3.03$ ) showing the load $P^{*}$ vs. dimensionless displacement during the loading phase of the first cycle for a rough dimple dimple. Therefore, the relative contribution of the asperities to the total displacement, which in Fig. 13 is still larger than that of the sphere bulk, is not as dominant as in the case of the rough dimple shown in Fig. 12.

To simulate the effect of asperity flattening after a number of load-unload cycles (see Figs. 6, 7, 8), a low value of the plasticity index $\psi=0.5$ was assumed. This $\psi$ value corresponds to $\sigma=2 \mathrm{~nm}$. The value of $\sigma$ was obtained from surface roughness measurements of a laserpolished dimple after 50 load-unload cycles. In this case, $\omega_{\mathrm{c}}^{*}=66.5$ and $P_{\mathrm{t}}^{*}=0.014$. The theoretical results are compared in Fig. 14 with the experimental ones for the 50th loading of a laser-polished dimple. As would be expected from the very small transition load, the contribution of the contacting asperities to the total displacement is smaller than the contribution of the bulk of the sphere [31] over most of the loading range.

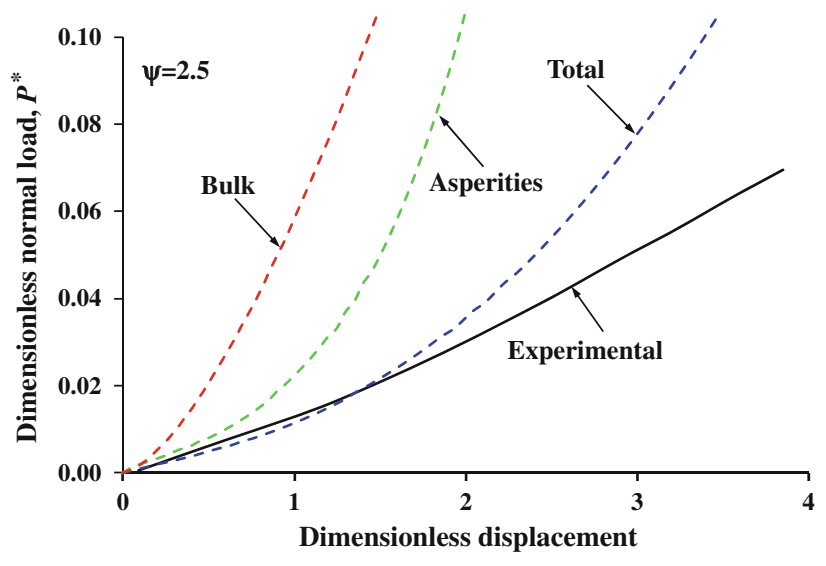

Fig. 13 A comparison of experimental results (solid line) and theoretical predictions (dashed lines $\psi=2.5, \omega_{\mathrm{c}}^{*}=4.43, P_{\mathrm{t}}^{*}=0.25$ ) showing the load $P^{*}$ vs. dimensionless displacement during the loading phase of the first cycle for a laser-polished dimple

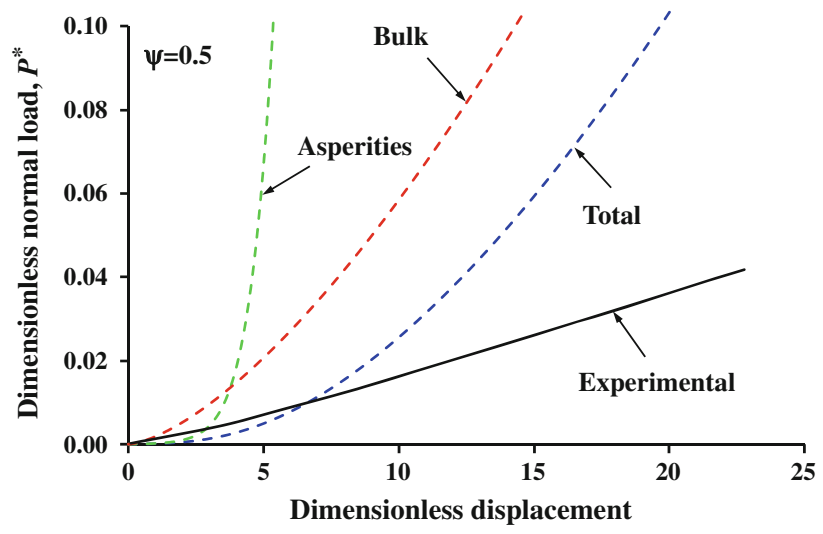

Fig. 14 A comparison of experimental results (solid line) and theoretical prediction (dashed lines $\psi=0.5, \quad \omega_{\mathrm{c}}^{*}=66.5, \quad P_{\mathrm{t}}^{*}=$ $0.014)$, showing the load $P^{*}$ vs. dimensionless displacement during the loading phase of the 50th cycle for a laser-polished dimple 


\section{Conclusion}

The effect of asperity flattening on the displacement of a rough spherical contact during repetitive load-cycling was investigated experimentally. Tests were performed with dimples of a hard disk drive suspension that were pressed against a sapphire flat using a modified nano-indenter test rig. Two different types of dimples, a rough dimple and a smoother laser-polished dimple were studied.

Both the dimple types exhibit substantial hysteresis of the load-displacement curve during the first load-unload cycles and a tendency toward reduced plastic deformation with subsequent load-unload cycles (elastic shakedown). The values of the maximum displacement, the residual displacement, and the energy dissipation of the smoother laser-polished dimple were smaller than their corresponding values for a rough dimple. Surface roughness measurements, before and after tests, showed substantial flattening of contacting asperities. This flattening, which results in a smoother contact area seems to be the main cause for the observed tendency to elastic shakedown.

A good correlation between the experimental results and the theoretical prediction of a recent model for rough spherical contact was found. The model clearly shows that for rougher surfaces the total displacement is mainly contributed by the contacting asperities while for smoother surfaces the main contribution comes from the bulk displacement.

Acknowledgments We would like to thank Mr. Edward B. Fanslau, and Hanya-san of NHK International Corporation for their interest and support of this research. We would also like to thank $\mathrm{Mr}$. J. McGuire and M. Berg from Hysitron Inc. for providing the plastic holders for the nano-indenter test rig, and help with providing photographs of the test equipment. L. Li thanks the China Scholarship Council (CSC) and Prof. G. Zhang from Harbin Institute of Technology, for supporting his Ph.D. studies at UCSD.

Open Access This article is distributed under the terms of the Creative Commons Attribution Noncommercial License which permits any noncommercial use, distribution, and reproduction in any medium, provided the original author(s) and source are credited.

\section{References}

1. McGruer, N.E., Adams, G.G., Chen, L., Guo, Z.J., Du, Y.: Mechanical, thermal, and material influences on ohmic-contacttype MEMS switch operation. In: MEMS 2006, Istanbul, Turkey, pp. 230-233 (2006)

2. Chung, K.H., Kim, D.E.: Fundamental investigation of micro wear rate using an atomic force microscope. Tribol. Lett. 15, 135-144 (2003)

3. Suh, A.Y., Polycarpou, A.A.: Adhesive contact modeling for sub5-nm ultralow flying magnetic storage head-disk interfaces including roughness effects. J. Appl. Phys. 97, 104328 (2005)

4. Li, L., Etsion, I., Fanslau, E.B., Talke, F.E.: An analysis of the dimple/gimbal contact in a hard disk drive suspension.
In: Proceedings of IIP/ISPS Joint MIPE 2009, Yokohama, Japan, pp. 105-106 (2009)

5. Lee, D.Y., Hwang, J., Bae, G.N.: Effect of disk rotational speed on contamination particles generated. Microsyst. Technol. 10, 103-108 (2004)

6. Kogut, L., Etsion, I.: Elastic-plastic contact analysis of a sphere and a rigid flat. J. Appl. Mech. Trans. ASME 69, 657-662 (2002)

7. Jackson, R.L., Green, I.: A finite element study of elasto-plastic hemispherical contact against a rigid flat. J. Tribol. Trans. ASME 127, 343-354 (2005)

8. Brizmer, V., Kligerman, Y., Etsion, I.: The effect of contact conditions and material properties on the elasticity terminus of a spherical contact. Int. J. Solids Struct. 43, 5736-5749 (2006)

9. Brizmer, V., Zait, Y., Etsion, I.: The effect of contact conditions and material properties on elastic-plastic spherical contact. J. Mech. Mater. Struct. 1, 865-879 (2006)

10. Cohen, D., Kligerman, Y., Etsion, I.: The effect of surface roughness on static friction and junction growth of an elastic-plastic spherical contact. J. Tribol. Trans. ASME 131, 021404 (2009)

11. Kagami, J., Yamada, K., Hatazawa, T.: Contact between a sphere and rough plates. Wear 87, 93-105 (1983)

12. Wang, F.S., Block, J.M., Chen, W.W., Martini, A., Zhou, K., Keer, L.M., Wang, Q.J.: A multilevel model for elastic-plastic contact between a sphere and a flat rough surface. J. Tribol. Trans. ASME 131, 021409 (2009)

13. Parker, R., Hatch, D.: The static coefficient of friction and the area of contact. Proc. Phys. Soc. Lond. B63 185 (1950)

14. Chaudhri, M., Yoffe, E.: The area of contact between a small sphere and a flat surface. Philos. Mag. A 44, 667-675 (1981)

15. Ovcharenko, A., Halperin, G., Verberne, G., Etsion, I.: In situ investigation of the contact area in elastic-plastic spherical contact during loading-unloading. Tribol. Lett. 25(2), 153-160 (2007)

16. Aymerich, F., Pau, M.: Assessment of nominal contact area parameters by means of ultrasonic waves. J. Tribol. Trans. ASME 126, 639-645 (2004)

17. Etsion, I., Levinson, O., Halperin, G., Varenberg, M.: Experimental investigation of the elastic-plastic contact area and static friction of a sphere on flat. J. Tribol. Trans. ASME 127, 47-50 (2005)

18. Jamari, J., Schipper, D.J.: Experimental investigation of fully plastic contact of a sphere against a hard flat. J. Tribol. Trans. ASME 128, 230-235 (2006)

19. Jamari, J., Schipper, D.J.: Deformation due to contact between a rough surface and a smooth ball. Wear 262, 138-145 (2007)

20. Lo, S.W., Yang, T., Shih, Z.M., Lin, S.C.: Effects of surface roughening on asperity flattening. Tribol. Lett. 35, 67-75 (2009)

21. Mesarovic, S.D., Johnson, K.L.: Adhesive contact of elasticplastic spheres. J. Mech. Phys. Solids 48, 2009-2033 (2000)

22. Li, L.Y., Wu, C.Y., Thornton, C.: A theoretical model for the contact of elasto-plastic bodies. Proc. Inst. Mech. Eng. C 216, 421-431 (2002)

23. Etsion, I., Kligerman, Y., Kadin, Y.: Unloading of an elasticplastic loaded spherical contact. Int. J. Solids Struct. 42, 3716-3729 (2005)

24. Kadin, Y., Kligerman, Y., Etsion, I.: Unloading of an elasticplastic contact of rough surfaces. J. Mech. Phys. Solids 54, 2652-2674 (2006)

25. Kadin, Y., Kligerman, Y., Etsion, I.: Multiple loading-unloading of an elastic-plastic spherical contact. Int. J. Solids Struct. 43, 7119-7127 (2006)

26. Qian, L., Zhang, J., Zhou, Z.: Radial nanofretting behavior of four typical structural materials in MEMS under high loads. Tribol. Int. 42, 1242-1249 (2009)

27. Kogut, L., Komvopoulos, K.: Analysis of the spherical indentation cycle for elastic-perfectly plastic solids. J. Mater. Res. 19, 3641-3653 (2004) 
28. König, J.A.: Shakedown of elastic-plastic structures. Elsevier, Amsterdam, New York, US (1987)

29. Greenwood, J.A., Williamson J.B.P.: Contact of nominally flat surfaces. Proc. R. Soc. Lond. A, 300-319 (1966)

30. Li, L., Etsion, I., Ovcharenko, A., Talke, F.E.: The onset of plastic yielding in a spherical shell compressed by a rigid flat. J. Appl. Mech. Trans. ASME. doi:10.1115/1.4001994 (In print)
31. Li, L., Etsion, I., Talke, F.E.: Elastic-plastic spherical contact modeling including roughness effects (under review)

32. McCool, J.I.: Comparison of models for the contact of rough surfaces. Wear 107, 37-60 (1986)

33. Thomas, T., Rosén, B.: Determination of the optimum sampling interval for rough contact mechanics. Tribol. Int. 33, 601-610 (2000) 